\title{
Quality of Life After Surgical Treatment of Early Barrett's Cancer: A Prospective Comparison of the Ivor-Lewis ResectionVersus the Modified Merendino Resection. A Statistical Hint: Reply
}

\author{
Christina Zapletal · Dietmar Lorenz
}

Published online: 5 September 2014

(c) Société Internationale de Chirurgie 2014

\section{Sir}

We thank Dr. Mangano and his colleagues for their recognition of our modest piece of work and for their valuable and important comments on biostatistics.

Generally, it is indeed difficult to draw conclusions from nonsignificant statistical tests as they are designed to control the alpha error, not the beta error. The latter, or the statistical power (which is 1-beta), can only be calculated a priori, not post hoc. The statistical power, as defined a priori, corresponds to the probability to detect a given true difference with the calculated sample size. Because our study was not designed as a randomized controlled trial (RCT), we did not define a primary endpoint, which is the precondition of sample size and power calculations.

It is not true, however, that no conclusions can be drawn from significant statistical tests without a power analysis.
As already noted, these tests are designed to control the alpha error, if significant. They do this irrespective of the beta error. Our conclusions were largely based on direct comparisons between the Merendino procedure (MER) and transthoracic esophageal resection (TER), where we found that TER was superior.

Most nonsignificant items also point in the same direction. Even with a larger sample and a higher probability of detecting hypothetical differences (=power), the effects are highly unlikely to change direction. If these effects were used as a basis for sample size calculations of a future $\mathrm{RCT}$, the resulting trial could only be designed to prove the superiority of TER. Given the claim of MER to buy quality of life, such a trial may raise ethical concerns.
C. Zapletal $(\bowtie)$

Department of Surgery, Dr. Horst Schmidt Klinik, Wiesbaden,

Germany

e-mail: czap@gmx.net

D. Lorenz

Department of Surgery, Sana Klinikum Offenbach, Offenbach, Germany 